\title{
Fragmentation functions of pions and kaons in the nonlocal chi- ral quark model
}

\author{
Chung Wen Kao ${ }^{1, a}$, Dong Jing Yang ${ }^{2}$, Fu Jiun Jiang ${ }^{2}$, and Seng-il Nam ${ }^{3}$ \\ ${ }^{1}$ Department of Chung Yuan Christian University,, Chung-Li 32023, Taiwan \\ ${ }^{2}$ Department of Physics, National Taiwan Normal University, Taipei 11677, Taiwan \\ ${ }^{3}$ School of Physics, Korea Institute for Advanced Study (KIAS), Seoul 130-722, Korea
}

\begin{abstract}
We investigate the unpolarized pion and kaon fragmentation functions using the nonlocal chiral-quark model. In this model the interactions between the quarks and pseudoscalar mesons is manifested nonlocally. In addition, the explicit flavor SU(3) symmetry breaking effect is taken into account in terms of the current quark masses. The results of our model are evaluated to higher $Q^{2}$ value $Q^{2}=4 \mathrm{GeV}^{2}$ by the DGLAP evolution. Then we compare them with the empirical parametrizations. We find that our results are in relatively good agreement with the empirical parametrizations and the other theoretical estimations.
\end{abstract}

\section{Introduction}

The unpolarized fragmentation function $D_{q}^{h}(z)$ represents the probability for a quark $q$ to emit a hadron $h$ with the light-cone momentum fraction $z$. It can be written with the light-cone coordinate as follows :

$$
D_{q}^{h}(z, \mu)=\pi z^{2} \int_{0}^{\infty} D_{q}^{h}\left(z, \boldsymbol{k}_{\perp}, \mu\right) d \boldsymbol{k}_{\perp}^{2}, D_{q}^{h}\left(z, \boldsymbol{k}_{T}^{2}, \mu\right)=\left.\frac{1}{4 z} \int d k^{+} \operatorname{Tr}\left[\Delta(k, p, \mu) \gamma^{-}\right]\right|_{z k^{-}=p^{-}} .
$$

Here, $k_{ \pm}=\left(k_{0} \pm k_{3}\right) / \sqrt{2}$ and the correlation $\Delta(k, p, \mu)$ is defined as

$$
\Delta(k, p, \mu)=\sum_{X} \int \frac{d^{4} \xi}{(2 \pi)^{4}} e^{+i k \cdot \xi}\langle 0|\psi(\xi)| h, X\rangle\langle h, X|\psi(0)| 0\rangle,
$$

where $k, p$ indicate the four-momenta for the initial quark and fragmented hadron, respectively. In addition, $z$ is the light-cone momentum fraction possessed by the hadron and $\mu$ denotes a renormalization scale at which the fragmentation process is computed. Furthermore, $\boldsymbol{k}_{\perp}$ is the transverse momentum of the initial quark and $\boldsymbol{k}_{T}=\boldsymbol{k}-\left[(\boldsymbol{k} \cdot \boldsymbol{p}) /|\boldsymbol{p}|^{2}\right] \boldsymbol{p}$ is the transverse momentum of the initial quark with respect to the direction of the momentum of the produced hadron. Finally $X$ appearing above stands for intermediate quarks. Notice all the calculations done here are carried out in the frame where the $z$-axis is chosen to be the direction of $\boldsymbol{k}$. Consequently one has $\boldsymbol{k}_{\perp}=0$ and $\boldsymbol{k}_{T} \neq 0$ in this frame. Empirically, information of $D_{q}^{h}(z)$ has to be extracted from the available high-energy lepton-scattering

\footnotetext{
a e-mail: cwkao@cycu.edu.tw
} 
data by global analysis with appropriate parametrizations satisfying certain constraints. For brevity, we will simply refer to the unpolarized fragmentation functions as the fragmentation functions from now on.

In Ref. [1], the Nambu-Jona-Lasinio (NJL) model has been used to calculate the elementary fragmentation functions. We have also employed the nonlocal chiral quark model (NLChQM) with the explicit flavor SU(3) symmetry breaking to calculate the elementary fragmentation functions [2, 3]. These instanton-motivated approaches were used for computing the quark distribution amplitudes, manifesting the nonlocal quark-pseudoscalar (PS) meson interactions. The elementary fragmentation functions are the functions in Eq. (2) with the following approximation:

$$
\sum_{X}|h, X\rangle\langle h, X|\approx| h=q \bar{Q}, X=Q\rangle\langle h=q \bar{Q}, X=Q| .
$$

Here $h$ denotes the PS meson. In other words, we just calculate the one-step fragmentation process: $q(k) \rightarrow h(p)+Q(k-p)$. Here the PS meson $h$ consists of the quark $q$ and the anti-quark $\bar{Q}$. In Refs. [4-6], the NJL model has been applied for the fragmentation functions including the quark-jets and resonances. The approach is actually applicable for any effective model. Here we present the result of NLChQM including the quark-jet contribution.

\section{Inclusion of multi-jets contribution}

To calculate the quark-jet contribution to the fragmentation functions within our model, we follow the approach in Refs. [1, 4-6]. The elementary fragmentation functions $\hat{d}_{q}^{h}(z)$ are re-defined as follows,

$$
\sum_{h} \int \hat{d}_{q}^{h}(z)=\sum_{Q} \int \hat{d}_{q}^{Q}(z) d z=1,
$$

where the complementary fragmentation functions $\hat{d}_{q}^{Q}(z)$ are given by

$$
\hat{d}_{q}^{Q}(z)=\hat{d}_{q}^{h}(1-z) . \quad h=q \bar{Q} .
$$

The fragmentation functions $D_{q}^{h}(z)$ should satisfy the following integral equation:

$$
D_{q}^{h}(z) d z=\hat{d}_{q}^{h}(z) d z+\sum_{Q} \int_{z}^{1} d y \hat{d}_{q}^{Q}(y) D_{Q}^{h}\left(\frac{z}{y}\right) \frac{d z}{y} .
$$

Note that $D_{q}^{h}(z) d z$ in Eq. (6) has a physical interpretation: $D_{q}^{h}(z) d z$ is the probability for a quark $q$ to emit a hadron which carries the light-cone momentum fraction from $z$ to $z+d z$. $\hat{d}_{q}^{Q}(y) d y$ is the probability for a quark $q$ to emit a hadron with flavor composition $q \bar{Q}$ and a quark $Q$ with the lightcone momentum fraction from $y$ to $y+d y$, at one stop. Eq. (6) actually describes a fragmentation cascade process of hadron emissions of a single quark.

According to charge conjugation and isospin symmetry, there are only 11 independent elementary fragmentation functions. Notice among these only four of them are not zero. We call these direct fragmentation functions:

$$
\begin{aligned}
& D_{u}^{\pi^{+}}(z)=D_{d}^{\pi^{-}}(z)=D_{\bar{u}}^{\pi^{-}}(z)=D_{\bar{d}}^{\pi^{+}}(z), \quad D_{u}^{\pi^{0}}(z)=D_{d}^{\pi^{0}}(z)=D_{\bar{u}}^{\pi^{0}}(z)=D_{\bar{d}}^{\pi^{0}}(z) \\
& D_{u}^{K^{+}}(z)=D_{d}^{K^{0}}(z)=D_{\bar{u}}^{K^{-}}(z)=D_{\bar{d}}^{\overline{K^{0}}}(z), \quad D_{s}^{K^{-}}(z)=D_{s}^{\overline{K^{0}}}(z)=D_{\bar{s}}^{K^{+}}(z)=D_{\bar{s}}^{K^{0}}(z)
\end{aligned}
$$



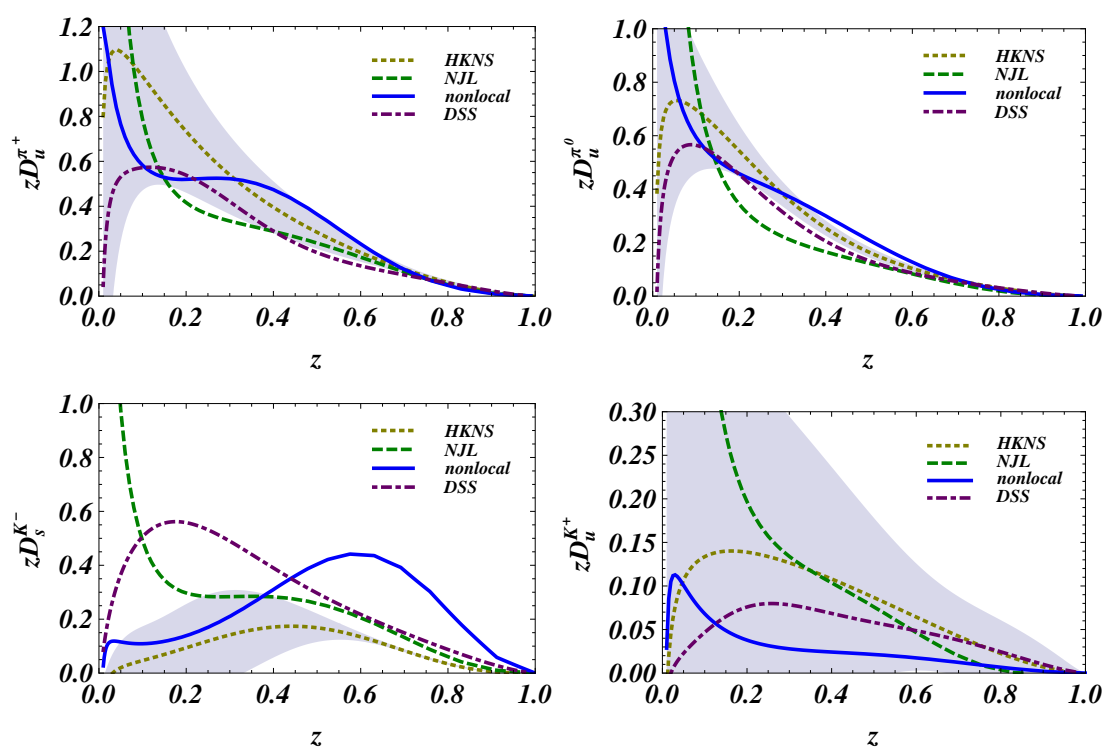

Figure 1. The fragmentation functions $z D_{u}^{\pi^{0}}(z)$ (upper panel, right) and $z D_{u}^{\pi^{+}}(z)$ (upper panel, left), $z D_{u}^{K^{+}}(z$ ) (bottom panel, right) and $z D_{s}^{K^{-}}(z)$ (bottom panel, left). The uncertainty bands are according to HKNS parameterizations.

The other ones are called indirect fragmentation functions listed as follows:

$$
\begin{aligned}
& D_{u}^{\pi^{-}}(z)=D_{d}^{\pi^{+}}(z)=D_{\bar{u}}^{\pi^{+}}(z)=D_{\bar{d}}^{\pi^{-}}(z), D_{u}^{K^{-}}(z)=D_{d}^{\overline{K^{0}}}(z)=D_{\bar{u}}^{K^{+}}(z)=D_{\bar{d}}^{K^{0}}(z), \\
& D_{u}^{K^{0}}(z)=D_{d}^{K^{+}}(z)=D_{\bar{u}}^{\overline{K^{0}}}(z)=D_{\bar{d}}^{K^{-}}(z), D_{u}^{\overline{K^{0}}}(z)=D_{d}^{K^{-}}(z)=D_{\bar{u}}^{K^{0}}(z)=D_{\bar{d}}^{K^{+}}(z), \\
& D_{s}^{K^{+}}(z)=D_{s}^{K^{0}}(z)=D_{\bar{s}}^{K^{-}}(z)=D_{\bar{s}}^{K^{0}}(z), D_{s}^{\pi^{+}}(z)=D_{s}^{\pi^{-}}(z)=D_{\bar{s}}^{\pi^{-}}(z)=D_{\bar{s}}^{K^{+}}(z), D_{s}^{\pi^{0}}(z)=D_{\bar{s}}^{\pi^{0}}(z) .
\end{aligned}
$$

\section{Results}

We present our results at $Q^{2}=4 \mathrm{GeV}^{2}$ and compare them with the empirical parametrizations and the NJL-jet model results. We employ QCDNUM17 [7] to evolve our results from $Q^{2}=0.36 \mathrm{GeV}^{2}$ to $Q^{2}=4 \mathrm{GeV}^{2}$. Since $D_{u}^{\pi^{+}}(z)$ is the most pronounced process, therefore, the initial momentum for evolution is determined by a reasonable agreement between our evolution result of $D_{u}^{\pi^{+}}(z)$ with two empirical parameterizations, namely the HKNS parametrization [8] and the DSS parametrization [9]. These two empirical parameterizations are used for comparison of other fragmentation functions as well. Our result shows a good agreement with those parametrizations [10].

\section{References}

[1] T. Ito, W. Bentz, I. -Ch. Cloet, A. W. Thomas and K. Yazaki, Phys. Rev. D 80, 074008 (2009).

[2] S. i. Nam and C. W. Kao, Phys. Rev. D 85, 034023 (2012).

[3] S. i. Nam and C. W. Kao, Phys. Rev. D 85, 094023 (2012)

[4] H. H. Matevosyan, A. W. Thomas and W. Bentz, Phys. Rev. D 83, 074003 (2011) 
EPJ Web of Conferences
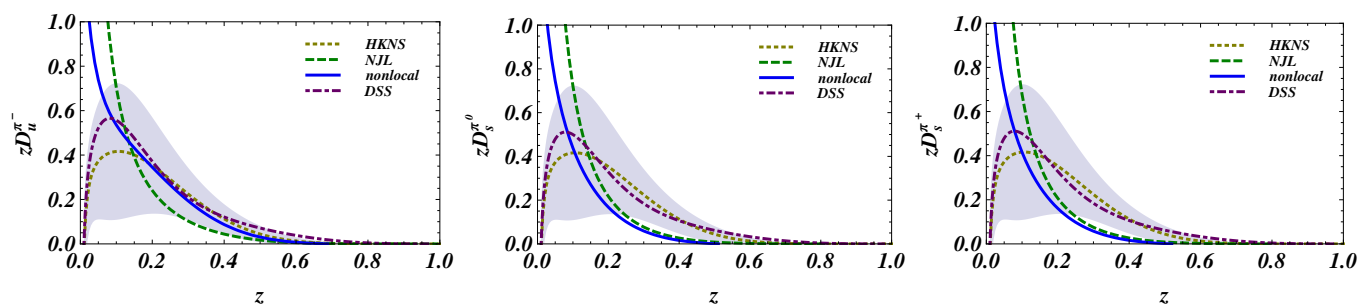

Figure 2. The fragmentation functions $z D_{u}^{\pi^{-}}(z)$ (left) and $z D_{s}^{\pi^{0}}(z)$ (middle) and $z D_{s}^{\pi^{+}}(z)$ (right). The dashed lines denote the result of NJL model. The uncertainty bands are according to the HKNS parameterizations.
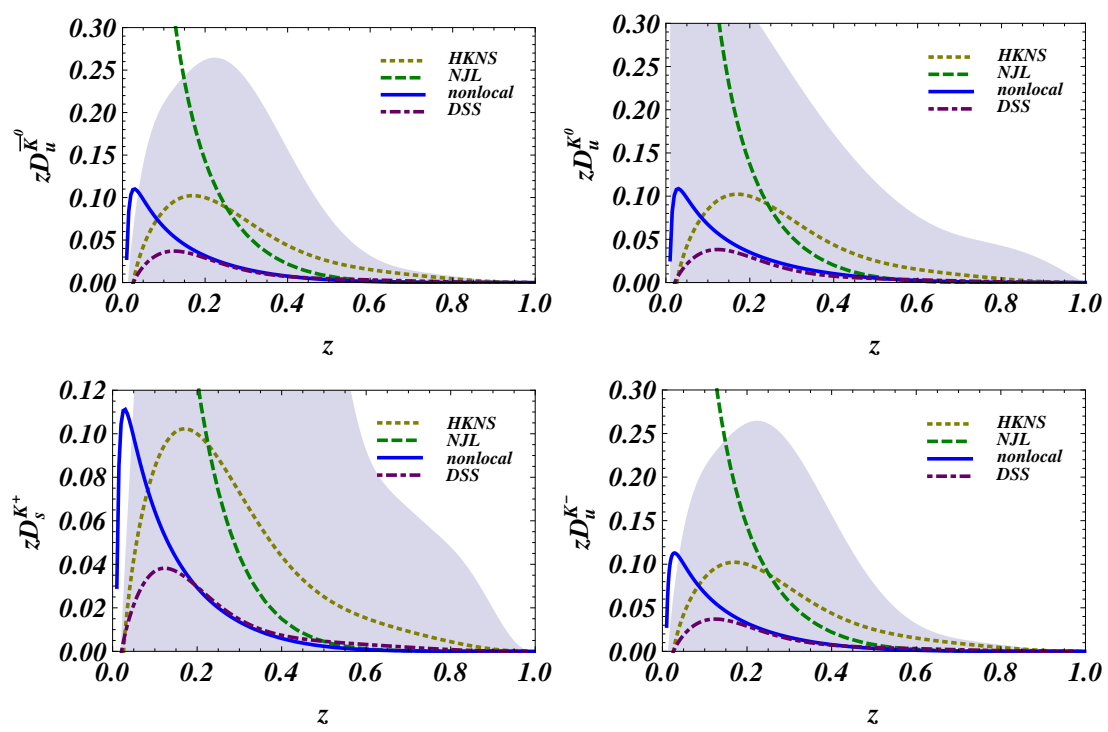

Figure 3. The fragmentation functions $z D_{u}^{K^{0}}$ (upper panel, right) and $z D_{u}^{\bar{K}^{0}}$ (upper panel, left), $z D_{u}^{K^{-}}$(bottom panel, right), and $z D_{s}^{K^{+}}$(bottom panel, left). The uncertainty bands are according to the HKNS parameterizations.

[5] H. H. Matevosyan, A. W. Thomas and W. Bentz, Phys. Rev. D 83, 114010 (2011).

[6] H. H. Matevosyan, W. Bentz, I. C. Cloet and A. W. Thomas, Phys. Rev. D 85, 014021 (2012).

[7] QCDNUM17, http://www.nikhef.nl/user/h24/qcdnum.

[8] M. Hirai, S. Kumano, T. H. Nagai and K. Sudoh, Phys. Rev. D 75, 094009 (2007).

[9] D. de Florian, R. Sassot and M. Stratmann, Phys. Rev. D 75, 114010 (2007).

[10] D. J. Yang, F. J. Jiang, C. W. Kao and S. i. Nam, Phys. Rev. D 87, 094007 (2013). 